\title{
The influence of light intensity and photoperiod on duckweed biomass and starch accumulation for bioethanol production
}

\author{
Yehu Yin ${ }^{\mathrm{a}, \mathrm{b}}$, Changjiang Yu ${ }^{\mathrm{a}}$, Li Yu ${ }^{\mathrm{a}}$, Jinshan Zhao ${ }^{\mathrm{c}}$, Changjiang Sun ${ }^{\mathrm{d}}$, Yubin Ma ${ }^{\mathrm{a}, *}$, Gongke Zhou ${ }^{\mathrm{a}}$ \\ ${ }^{a}$ Key Laboratory of Biofuels, Shandong Provincial Key Laboratory of Energy Genetics, Qingdao Institute of Bioenergy and Bioprocess Technology, Chinese Academy of Sciences, \\ Qingdao 266101, PR China \\ ${ }^{\mathrm{b}}$ University of Chinese Academy of Sciences, Beijing 100049, PR China \\ ' Qingdao Institute of Animal Sciences, Qingdao 266100, PR China \\ ${ }^{\mathrm{d}}$ Beijing Risun Chemical Industry Technology Research Institute Co. Ltd, Beijing 100070, PR China
}

\section{H I G H L I G H T S}

- The impact of light condition on duckweed biomass and starch accumulation was investigated.

- Light intensity and photoperiod had a significant effect on biomass and starch production.

- $110 \mu \mathrm{mol} \mathrm{m}{ }^{-2} \mathrm{~s}^{-1}$ was the best light condition for duckweed biomass and starch accumulation.

- The results suggested high light induction was an effective method for starch accumulation.

- This study provides optimized light conditions for future industrial duckweed cultivation.

\section{A R T I C L E I N F O}

\section{Article history:}

Received 27 January 2015

Received in revised form 20 March 2015

Accepted 21 March 2015

Available online 27 March 2015

\section{Keywords:}

Duckweed

Lemna aequinoctialis

Light intensity

Photoperiod

Starch production

\begin{abstract}
A B S T R A C T
Duckweed has been considered as a valuable feedstock for bioethanol production due to its high biomass and starch production. To investigate the effects of light conditions on duckweed biomass and starch production, Lemna aequinoctialis 6000 was cultivated at different photoperiods $(12: 12,16: 8$ and $24: 0 \mathrm{~h})$ and light intensities $\left(20,50,80,110,200\right.$ and $\left.400 \mu \mathrm{mol} \mathrm{m}^{-2} \mathrm{~s}^{-1}\right)$. The results showed that the duckweed biomass and starch production was increased with increasing light intensity and photoperiod except at 200 and $400 \mu \mathrm{mol} \mathrm{m}^{-2} \mathrm{~s}^{-1}$. Considering the light cost, $110 \mu \mathrm{mol} \mathrm{m}^{-2} \mathrm{~s}^{-1}$ was optimum light condition for starch accumulation with the highest maximum growth rate, biomass and starch production of $8.90 \mathrm{~g} \mathrm{~m}^{-2}$ day $^{-1}, 233.25 \mathrm{~g} \mathrm{~m}^{-2}$ and $98.70 \mathrm{~g} \mathrm{~m}^{-2}$, respectively. Moreover, the results suggested that high light induction was a promising method for duckweed starch accumulation. This study provides optimized light conditions for future industrial large-scale duckweed cultivation.
\end{abstract}

(C) 2015 Elsevier Ltd. All rights reserved.

\section{Introduction}

Nowadays, the energy crisis and environmental problems caused by the consumption of fossil fuels have attracted much more attention worldwide. The exploration for alternative energy sources has become an increasingly urgent requirement. Biorenewable energy, especially bioethanol and biodiesel, has been considered as an important alternative energy. Bioethanol is an alcohol made by sugar fermentation and can be used as liquid fuel and gasoline mixed agent to enhance oxygen content and reduce

* Corresponding author at: Qingdao Institute of Bioenergy and Bioprocess Technology, Chinese Academy of Sciences, No. 189 Songling Road, Laoshan District, Qingdao 266101, Shandong, China. Tel.: +86 53280662731 ; fax: +86 53280662778.

E-mail addresses: mayb@qibebt.ac.cn (Y. Ma),zhougk@qibebt.ac.cn (G. Zhou). emissions (Sánchez and Cardona, 2008). Most ethanol fuel is produced using corn as feedstock. However, using corn for ethanol production is inevitably competing for limited cropland for food/ feed production (Endo et al., 2008). Significantly, lignocellulosic biomass is of great potential source for ethanol production because the material is abundant in the world. However, conversion of lignocellulosic biomass to bioethanol is difficult and much more expensive due to the tight structure of the biomass (Sarkar et al. 2012). Therefore, there is great interest in exploring novel starch sources that do not necessarily compete for cropland and can be digested much easier than lignocellulosic biomass to make bioethanol production more sustainable. Recently, duckweed has drawn increasing attention for bioethanol production due to its high biomass and starch production (Xu et al., 2011, 2012; Cui and Cheng, 2015). 
Duckweed, a small floating aquatic plant, belongs to the monocotyledonous family of Lemnaceae. The geographic ranges of duckweed span the entire globe and 37 species belonging to five genera (Lemna, Landoltia, Spirodela, Wolffia, and Wolffiella) have been identified so far (Appenroth et al., 2013). Duckweed grows faster than most other plants, and under ideal condition, some species can double their biomass every $16 \mathrm{~h}$ to $24 \mathrm{~h}$ (Peng et al, 2007). The duckweed starch content varies by species and growth conditions ranging from $3 \%$ to $75 \%$ of dry weight (Reid and Bieleski, 1970; Xu et al., 2012; Cui and Cheng, 2015). Duckweed starch can be readily converted to ethanol using the same protocol that used for corn starch (Ge et al., 2012). The ethanol yield of duckweed reached $6.42 \times 10^{3} \mathrm{~L} \mathrm{ha}^{-1}$, which was about $50 \%$ higher than that of maize-based ethanol production (Xu et al., 2011). In addition to high starch content, there are lower lignin ( $2 \%$ dry weight) and cellulose ( $\sim 10 \%$ dry weight) content in duckweed than that in terrestrial plants (Ge et al., 2012; Zhao et al., 2014), indicating that duckweed biomass can be hydrolyzed much easier and the biomass-to-ethanol conversion process is more cost-effective (Cheng and Stomp, 2009; Ge et al., 2012). Taken together, the characteristics of rapid growth, high starch content and high digestibility make duckweed an ideal feedstock for bioethanol production.

Starch accumulation is the key consideration for duckweed bioethanol production. As to the influence factors for starch accumulation, nutrient starvation especially nitrogen and phosphorus, sodium, abscisic acid and other chemical growth inhibitors had been widely investigated (McLaren and Smith, 1976; Thorsteinsson and Tillberg, 1987; Janas and Osiecka, 1995; Janas et al., 1998; Cheng and Stomp, 2009; Xu et al., 2011, 2012; Cui and Cheng, 2015). On the other hand, starch is the product from the photosynthesis, light is the only source of energy for photosynthesis, and also the direct source of energy for starch accumulation (Stitt and Zeeman, 2012). However, only a few reports had been focus on the effect of light condition on duckweed starch accumulation. Cui et al. (2011) determined the starch content variation at low light intensity $\left(40.5 \mu \mathrm{mol} \mathrm{m} \mathrm{m}^{-2} \mathrm{~s}^{-1}\right)$ under three photoperiod conditions (8:16, 12:12 and 16:8 h light:dark) after 6 days of cultivation. Zhao et al. (2014) measured the growth rate and starch content at 2000, 5000 and 10,000 lux after inoculation for 12 days. To the best of our knowledge, few systemic studies had been done on the effects of light conditions on duckweed starch accumulation.

Moreover, with the development of duckweed commercialization, large-scale industrial cultivation model will be popularized in duckweed cropping system. For example, Xu et al. (2012) suggested that novel cultivation reactors such as multilayer or pagoda-shaped structures would be applied in the future largescale duckweed cultivation. In order to promote duckweed commercialization, we have established duckweed multilayer pilot reactor and $1500 \mathrm{~m}^{2}$ duckweed large-scale multilayer cropping system using waste water (Unpublished results). Light condition is one of important factors for industrial cultivation model in the future, such as multilayer pilot reactors and duckweed multilayer cropping system. Therefore, it is necessary to study the effect of light condition on the duckweed biomass and starch accumulation systematically. Thus, in this study, we investigated the effects of different light intensities and different photoperiod regimes (exposure to light:dark cycles) on duckweed growth density, growth rate, biomass production, starch content and starch production. The aim of this study is to provide the possibility of improving bioethanol production through controlling light intensity and photoperiod for future industrial large-scale duckweed cultivation. Duckweed was cultivated for 39 days at six different light intensities $\left(20,50,80,110,200\right.$ and $\left.400 \mu \mathrm{mol} \mathrm{m}^{-2} \mathrm{~s}^{-1}\right)$ and three different photoperiod cycles (12:12, 16:8 and 24:0 h light:dark).

\section{Methods}

\subsection{Duckweed strain and culture method}

Lemna aequinoctialis 6000, which had the best ability to accumulate starch, was isolated from Lixian county in Hunan province by large-scale screening of more than 100 clones of duckweed distributed in 20 provinces in China (Yu et al., 2014, 2015). L. aequinoctialis 6000 was pre-cultured in a large tub $(60 \mathrm{~cm} \times 40 \mathrm{~cm} \times 10 \mathrm{~cm}$ ) for 2 weeks on $1 / 2$ Schenk-Hildebrandt medium (SH) (Schenk and Hildebrandt, 1972). The pH of the medium was adjusted to 5.80 . The tub was placed into a $23^{\circ} \mathrm{C}$ growth chamber with the photoperiod of $16: 8$ and light intensity of $80 \mu \mathrm{mol} \mathrm{m} \mathrm{m}^{-2} \mathrm{~s}^{-1}$ provided by wide spectrum fluorescent tubes.

The experiment was carried out using $750 \mathrm{~mL}$ plastic box $(12.5 \mathrm{~cm} \times 12.5 \mathrm{~cm} \times 4.2 \mathrm{~cm})$. Each box was filled with $500 \mathrm{~mL} \mathrm{1/}$ $2 \mathrm{SH}$ medium and inoculated with $2 \mathrm{~g}$ fresh duckweed to cover the entire water surface with a single layer of fronds. Duckweed was cultivated for 39 days at six different light intensities (20, 50, 80, 110, 200 and $400 \mu \mathrm{mol} \mathrm{m}^{-2} \mathrm{~s}^{-1}$ ) and three different photoperiod regimes $(12: 12,16: 8$ and $24: 0 \mathrm{~h}$ light:dark). The evaporated water was replenished with distilled water every day throughout the experiments. The light intensity was measured using a quantum photometer (LI-250A, LI-COR, USA). Each experiment was performed in three replicates for different light intensities and photoperiod cycles.

\subsection{Measurement of duckweed growth}

To investigate the duckweed growth, L. aequinoctialis 6000 was harvested at interval of 3 days from each box using a strainer to remove fronds from $20 \%$ of the surface area based on the previous report (Xu and Shen, 2011). The duckweed growth density was calculated based on the amount of duckweed harvested each time. To measure the wet weight, duckweed was rinsed with distilled water using a strainer. After free water stopped dripping, duckweed was blotted dry with paper towels, and then measured with a balance (Bergmann et al., 2000). To measure dry weight (DW), the fresh fronds were lyophilized using a freeze drier (ALPHA1-2LD PLUS, CHRIST, Germany) for 48 hours.

The logistic model was applied to describe the duckweed growth according to the research in other species (Ma et al., 2013):

$B_{t}=\frac{B_{f}}{1+\frac{B_{f}-B_{0}}{B_{0}} * \exp ^{\frac{-4 \mu_{\max }}{B_{f}}}}$

where $B_{0}$ and $B_{f}$ are the dry weight at zero and stationary phase, $t$ and $B_{t}$ are time and the corresponding dry weight, and $\mu_{\max }$ is the maximum population growth rate, which can thus be obtained after nonlinear-fitting with the above equation using the software origin 7.0 .

\subsection{Duckweed starch extraction and quantification}

The starch content was determined using a modified method (Smith and Zeeman, 2006). Briefly, $50 \mathrm{mg}$ frozen dry duckweed was grinded into powder by a multi-tube ball mill (Tissuelyser II, Qiagen, Germany). Then, $1.5 \mathrm{~mL} 80 \%$ ethanol was added. After incubating in $70^{\circ} \mathrm{C}$ for $15 \mathrm{~min}$, the sample was centrifuged for $5 \mathrm{~min}(12,000 \mathrm{~g})$ at room temperature and then the supernatant solution was discarded. This process was repeated for twice. After evaporating ethanol, transferred the precipitate to volumetric flask and added distilled water to $10 \mathrm{~mL}$, then taken $0.5 \mathrm{~mL}$ homogenate into $2 \mathrm{~mL}$ EP tube and heated in $100{ }^{\circ} \mathrm{C}$ for $10 \mathrm{~min}$. After cooling, $0.5 \mathrm{~mL} 200 \mathrm{mM}$ Na acetate (PH 4.8) mixed with $1 \mu \mathrm{L} \alpha$ amyloglucosidase (Sigma A7095) and $1 \mu \mathrm{L}$ a-amylase (Sigma 
A4582) were added. The mixture was incubated at $37^{\circ} \mathrm{C}$ for $4 \mathrm{~h}$. Then, the tube was centrifuged for $5 \mathrm{~min}$ at $12,000 \mathrm{~g}$. The glucose in the supernatant was the hydrolyzed production of duckweed starch. The glucose in the supernatant was analyzed by Waters HPLC system (Waters 2545, Waters Corp., USA) equipped with a Hypersil ODS-2 C18 column $(4.6 \mathrm{~mm} \times 250 \mathrm{~mm}$; Thermo, Thermo Corp., USA) and a 2489 Uv/Vis detector (Yu et al., 2014, 2015; Xiao et al., 2013; Zhao et al., 2014). The starch content was determined using the total glucose content (starch content $=$ glucose content $\times$ 0.909) (Zhang et al., 2011).

\section{Results and discussion}

\subsection{Effect of light intensity and photoperiod on duckweed growth}

To illustrate the effect of the light intensity and photoperiod on duckweed growth, L. aequinoctialis 6000 was cultivated for 39 days at three different photoperiods (12:12, 16:8 and 24:0 h light:dark) and six different light intensities (20, 50, 80, 110, 200 and $\left.400 \mu \mathrm{mol} \mathrm{m} \mathrm{m}^{-2} \mathrm{~s}^{-1}\right)$. The results showed that light intensity and photoperiod had a significant effect on duckweed growth. Fig. 1 illustrated the dynamic variation of duckweed growth density during cultivation under different light intensities and photoperiods. The results indicated that the growth density was increased in the initial cultivation, reaching the highest level and then slowly decreasing during the remaining days (Fig. 1). Furthermore, we found that the growth density was increased with increasing light intensity except at 200 and $400 \mu \mathrm{mol} \mathrm{m}{ }^{-2} \mathrm{~s}^{-1}$ under a photoperiod of 24:0 h light/dark cycle (Table 1 and Fig. 1 ). As to the influence of photoperiod, the results showed that the variation of growth density depended on the light intensity. At $200 \mu \mathrm{mol} \mathrm{m}^{-2} \mathrm{~s}^{-1}$ and lower light intensity $\left(20,50,80\right.$ and $\left.110 \mu \mathrm{mol} \mathrm{m} \mathrm{m}^{-2} \mathrm{~s}^{-1}\right)$, the growth density was increased with extending photoperiod. However, at light intensity of $400 \mu \mathrm{mol} \mathrm{m}{ }^{-2} \mathrm{~s}^{-1}$, extended photoperiod resulted in lower growth density (Table 1 and Fig. 1).

Growth density could reflect the dynamic variation of duckweed growth status during cultivation. However, biomass production is the key consideration in duckweed cultivation. In order to get the highest biomass production, the duckweed was harvested at interval of 3 days from each box using a strainer to remove fronds from $20 \%$ of the surface area as reported previously (Xu and Shen, 2011). The results showed that the duckweed biomass production was constantly increased during cultivation except for 200 and $400 \mu \mathrm{mol} \mathrm{m}^{-2} \mathrm{~s}^{-1}$ under a photoperiod of 24:0 h light/dark cycle (Fig. 2). Furthermore, we found that the effect of light intensity and photoperiod on biomass production was similar with the rule of growth density variation. Increased light intensity resulted in higher biomass production excluding 200 and $400 \mu \mathrm{mol} \mathrm{m}^{-2} \mathrm{~s}^{-1}$ under a photoperiod of 24:0 h light/dark cycle (Table 1 and Fig. 2). For the impact of photoperiod, the results showed that extended photoperiod resulted in higher biomass production at $200 \mu \mathrm{mol} \mathrm{m} \mathrm{m}^{-2} \mathrm{~s}^{-1}$ and lower light intensity. With increasing light intensity $\left(400 \mu \mathrm{mol} \mathrm{m}{ }^{-2} \mathrm{~s}^{-1}\right.$ ), the biomass production was decreased with extending photoperiod (Table 1 and Fig. 2).

To further characterize the duckweed growth, the duckweed maximum growth rate was estimated. The widely used logistic model with correlation coefficients above 0.995 was applied in this study (Ma et al., 2013). The results showed that increasing light intensity could improve the maximum growth rate except at 200 and $400 \mu \mathrm{mol} \mathrm{m}^{-2} \mathrm{~s}^{-1}$ under a photoperiod of 24:0 h light/dark cycle (Table 1). The highest maximum growth rate was $8.90 \mathrm{~g} \mathrm{~m}^{-2}$ day $^{-1}$ at $110 \mu \mathrm{mol} \mathrm{m} \mathrm{m}^{-2} \mathrm{~s}^{-1}$ under a photoperiod of 24:0 h light/dark cycle. Further, we found that the maximum growth rate at 200 and $400 \mu \mathrm{mol} \mathrm{m}{ }^{-2} \mathrm{~s}^{-1}$ under a photoperiod of
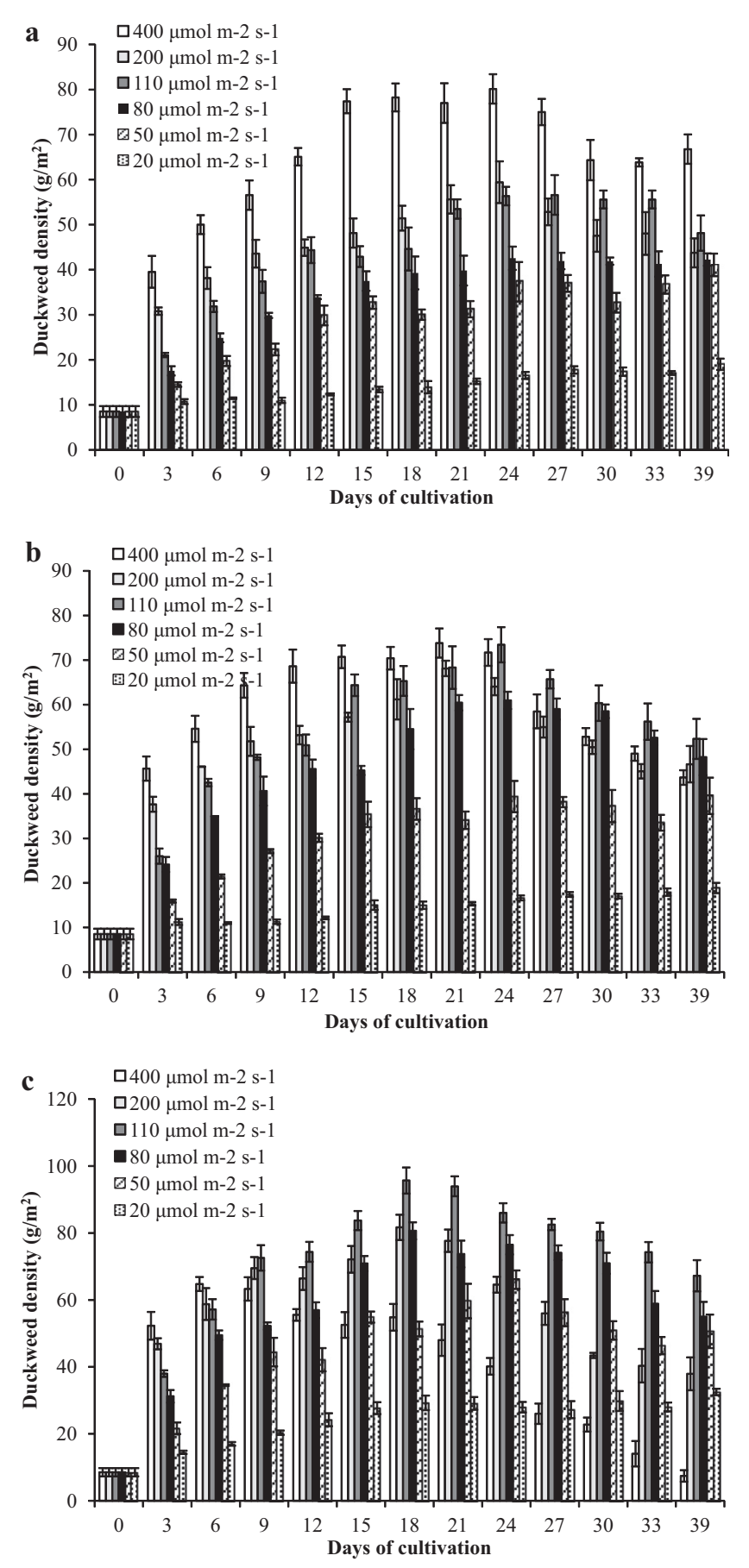

Fig. 1. Duckweed growth density under three different photoperiod cycles of 12:12 (a), 16:8 (b) and 24:0 (c) and six different light intensities (20, 50, 80, 110, 200 and $400 \mu \mathrm{mol} \mathrm{m}^{-2} \mathrm{~s}^{-1}$ ). Data are means of three repeated experiments and error bars indicate standard deviations.

24:0 h light/dark cycle was 8.01 and $8.61 \mathrm{~g} \mathrm{~m}^{-2}$ day $^{-1}$ which was not too lower compared to that in other condition. The reason might be that the growth density and biomass production in the initial 6 days was higher than that in other condition (Fig. 1).

Photosynthesis is the ultimate source of biomass production in plants. Plants efficiently gather light energy when light intensities are low, but they must also harmlessly dissipate excitation energy when photon absorption exceeds the capacity of the plants for photosynthesis (Niyogi, 1999). In high light, plants may absorb excess light energy, which can lead to the damage of the photosystem. High rates of damage (those that exceed the rate of repair) 
Table 1

Maximum growth density, maximum growth rate and maximum biomass production of duckweed cultivated at three photoperiod cycles and six light intensities.

\begin{tabular}{|c|c|c|c|c|}
\hline $\begin{array}{l}\text { Photoperiod (L:D } \\
\text { cycle) h }\end{array}$ & $\begin{array}{l}\text { Light intensity } \\
\left(\mu \mathrm{mol} \mathrm{m} \mathrm{m}^{-2} \mathrm{~s}^{-1}\right)\end{array}$ & $\begin{array}{l}\text { Maximum growth density } \\
\left(\mathrm{g} \mathrm{m}^{-2}\right)\end{array}$ & $\begin{array}{l}\text { Maximum growth rate } \\
\left(\mathrm{g} \mathrm{m}^{-2} \mathrm{day}^{-1}\right)\end{array}$ & $\begin{array}{l}\text { Maximum biomass production } \\
\left(\mathrm{g} \mathrm{m}^{-2}\right)\end{array}$ \\
\hline \multirow[t]{6}{*}{$12: 12$} & 400 & $80.13 \pm 3.27$ & 7.44 & $211.99 \pm 4.55$ \\
\hline & 200 & $59.43 \pm 4.62$ & 4.97 & $147.44 \pm 8.87$ \\
\hline & 110 & $56.60 \pm 4.41$ & 4.90 & $147.03 \pm 1.92$ \\
\hline & 80 & $42.56 \pm 5.73$ & 3.74 & $128.73 \pm 3.07$ \\
\hline & 50 & $41.06 \pm 2.51$ & 3.14 & $106.83 \pm 3.45$ \\
\hline & 20 & $19.01 \pm 1.24$ & 1.32 & $50.39 \pm 1.22$ \\
\hline \multirow[t]{6}{*}{$16: 8$} & 400 & $73.83 \pm 3.28$ & 7.19 & $179.71 \pm 2.71$ \\
\hline & 200 & $68.12 \pm 3.25$ & 5.88 & $164.61 \pm 5.87$ \\
\hline & 110 & $73.45 \pm 3.94$ & 6.72 & $177.30 \pm 3.68$ \\
\hline & 80 & $60.97 \pm 1.93$ & 5.44 & $155.57 \pm 4.24$ \\
\hline & 50 & $39.55 \pm 4.08$ & 3.37 & $109.34 \pm 4.05$ \\
\hline & 20 & $18.87 \pm 1.15$ & 1.34 & $50.91 \pm 1.43$ \\
\hline \multirow[t]{6}{*}{$24: 0$} & 400 & $64.76 \pm 2.09$ & 8.61 & $118.40 \pm 4.74$ \\
\hline & 200 & $81.68 \pm 3.75$ & 8.01 & $172.58 \pm 7.82$ \\
\hline & 110 & $95.68 \pm 3.89$ & 8.90 & $233.25 \pm 5.43$ \\
\hline & 80 & $80.64 \pm 2.54$ & 7.41 & $193.63 \pm 8.15$ \\
\hline & 50 & $66.04 \pm 2.76$ & 5.49 & $155.83 \pm 5.73$ \\
\hline & 20 & $32.50 \pm 0.97$ & 2.54 & $87.53 \pm 0.49$ \\
\hline
\end{tabular}

Data are means of three repeated experiments and error bars indicate standard deviations.

will cause reduction of photosynthetic efficiency (Im and Grossman, 2001). Besides light intensity, photoperiod is another important factor for plant growth. The effects of photoperiod on plants contain two aspects. Firstly, photoperiod is a signal for many processes in plant growth including the detection of the light signal in the leaves, the entrainment of circadian rhythms, and the production of a mobile signal which is transmitted throughout the plant (Jackson, 2009). Secondly, longer photoperiod can contribute to more light energy input and longer time of photosynthesis, resulting in more photosynthate production, thus leading to the enhancement of plant growth and development. In this study, at low light intensity $\left(20,50,80\right.$ and $\left.110 \mu \mathrm{mol} \mathrm{m}^{-2} \mathrm{~s}^{-1}\right)$, with the increased light intensity and photoperiod, the duckweed produced more photosynthate, thus leading to the higher biomass accumulation. However, at high light intensity especially $400 \mu \mathrm{mol} \mathrm{m}^{-2} \mathrm{~s}^{-1}$, the duckweed biomass accumulation was decreased with extending photoperiod, indicating that light intensity of $400 \mu \mathrm{mol} \mathrm{m}{ }^{-2}$ $\mathrm{s}^{-1}$ is high light for duckweed. With extending photoperiod, light damage rate exceed repair rate which may cause the reduction of photosynthetic efficiency, and then finally result in the decrease of biomass accumulation (Figs.1c and 2c). Besides light intensity, photoperiod also plays an important role in duckweed growth, further indicating that light intensity and photoperiod have crosscoupling effects on duckweed biomass accumulation. This study demonstrated the photoperiod effect on light energy input and photosynthate production in duckweed. As to the suggestion for future study, when light is one of major cost in industrial largescale duckweed cultivation, light intensity of $110 \mu \mathrm{mol} \mathrm{m}^{-2} \mathrm{~s}^{-1}$ under photoperiod of $24: 0 \mathrm{~h}$ light/dark cycle is the best choice for duckweed biomass accumulation. Much higher light intensity will result in lower economy based on our study. This study provides optimized light condition for duckweed large-scale multilayer cropping system. In the future study, the economic evaluation of the duckweed multiplayer cropping system will be carried out.

\subsection{Effect of light intensity and photoperiod on duckweed starch content}

Besides biomass production, starch accumulation is another key consideration in duckweed biofuel production. High-starch duckweed is a valuable feedstock for biofuel production. Light is the only source of energy for photosynthetic $\mathrm{CO}_{2}$ assimilation, and the direct source of energy for starch production in green plants (Stitt and Zeeman, 2012). To further investigate the effect of light intensity and photoperiod on starch accumulation, the starch content of duckweed cultivated at three different photoperiod cycles (12:12, 16:8 and 24:0 h light:dark) and six different light intensities $\left(20,50,80,110,200\right.$ and $\left.400 \mu \mathrm{mol} \mathrm{m}^{-2} \mathrm{~s}^{-1}\right)$ was determined in this study. The results showed that the duckweed starch content was increased with increasing light intensity and photoperiod (Fig. 3 and Table 2). Starch content variation during 39 days cultivation showed different pattern under different light intensity. Under low light intensity $\left(20 \mu \mathrm{mol} \mathrm{m}^{-2} \mathrm{~s}^{-1}\right)$, the duckweed starch content was decreased during the cultivation (Fig. 3). Under middle light intensity (50, 80 and $110 \mu \mathrm{mol} \mathrm{m}^{-2}$ $\mathrm{s}^{-1}$ ), the duckweed starch content was increased in the initial 3 days, decreasing in the following 18 days, and then increasing in the following cultivation days (Fig. 3). Under high intensity (200 and $400 \mu \mathrm{mol} \mathrm{m} \mathrm{m}^{-2} \mathrm{~s}^{-1}$ ), the duckweed starch content variation exhibited different pattern under three photoperiods. Under a photoperiod of 12:12 h (light:dark) cycle, the duckweed starch content was increased in the initial 3 days, decreasing in the following 12 days, and then increasing in the following cultivation days (Fig. 3a). With extending photoperiod (16:8 light:dark), starch content variation at $200 \mu \mathrm{mol} \mathrm{m}{ }^{-2} \mathrm{~s}^{-1}$ showed the similar pattern with the result in 12:12 h photoperiod. While, at light intensity of $400 \mu \mathrm{mol} \mathrm{m}{ }^{-2} \mathrm{~s}^{-1}$, starch content variation showed different pattern which consistently increased during the cultivation days (Fig. 3b). At the longest photoperiod (24:0 light:dark), the starch content was increased in the initial 6 days, decreasing in 9 days for $200 \mu \mathrm{mol} \mathrm{m} \mathrm{m}^{-2} \mathrm{~s}^{-1}$ and 15 days for $400 \mu \mathrm{mol} \mathrm{m} \mathrm{m}^{-2} \mathrm{~s}^{-1}$, and then increasing in the following cultivation days (Fig. 3c).

Starch is related with the balance of carbon source in green plants, which is mainly derived from photosynthesis and exhausted by respiration. When the import of carbon source, accumulated by photosynthesis or exogenous carbon sources, overweigh the export of carbon sources, such as respiration or reproduction, the starch begins to accumulate, and vice versa (Xu et al., 2012). In this study, increased light intensity and extended photoperiod in duckweed cultivation could enhance photosynthesis efficiency which resulted in more fluxes of carbon source into 

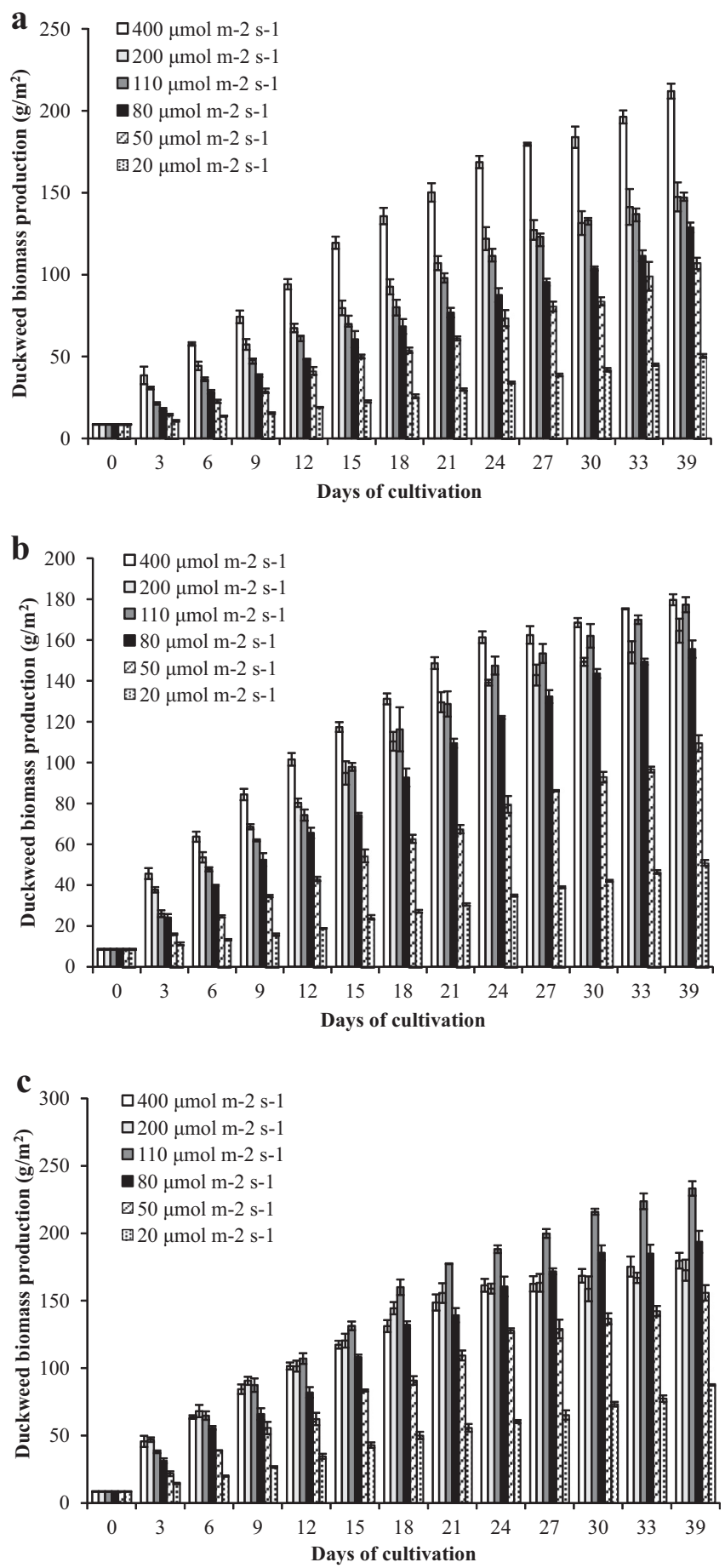

Fig. 2. Duckweed biomass production under three different photoperiod cycles of 12:12 (a), 16:8 (b) and 24:0 (c) and six different light intensities (20, 50, 80, 110, 200 and $400 \mu \mathrm{mol} \mathrm{m}^{-2} \mathrm{~s}^{-1}$ ). Data are means of three repeated experiments and error bars indicate standard deviations.

starch, and finally increased starch content in duckweed. The result was consistent with other reports in duckweed which increasing the light intensity or extending the photoperiod could increase the starch content (Cui et al., 2011; Zhao et al., 2014). Starch is an important photosynthate in duckweed, increased light intensity and photoperiod resulted in higher starch accumulation in duckweed in daytime, and finally, the higher stored starch ensured more substrates for metabolism and growth throughout the night. Duckweed higher starch accumulation may be one reason for its
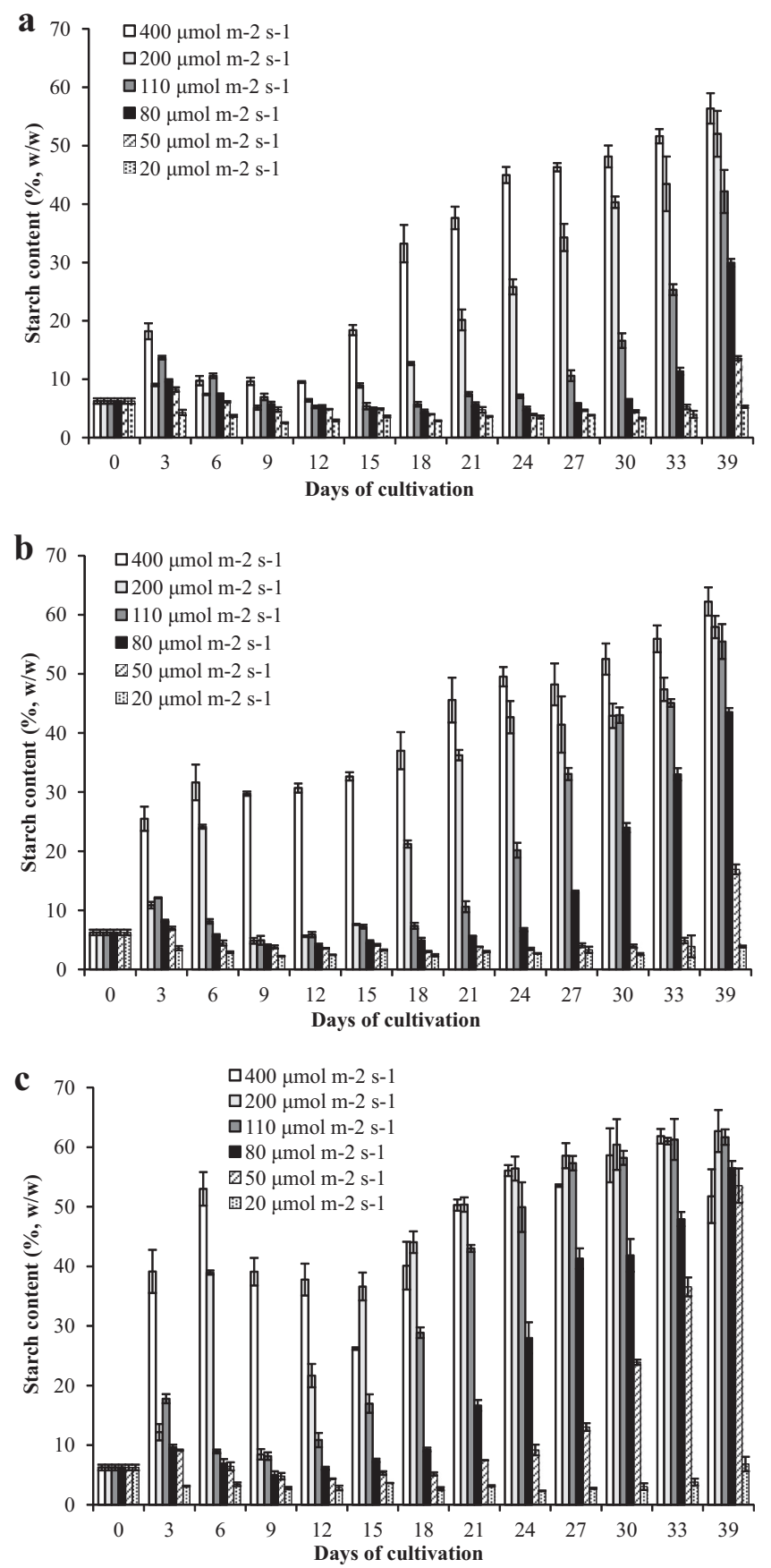

Fig. 3. Duckweed starch content under three different photoperiod cycles of 12:12 (a), 16:8 (b) and 24:0 (c) and six different light intensities (20,50, 80, 110, 200 and $400 \mu \mathrm{mol} \mathrm{m}^{-2} \mathrm{~s}^{-1}$ ). Data are means of three repeated experiments and error bars indicate standard deviations.

rapid growth than other plant in natural environment. Compared with other green plants, such as Arabidopsis, the model species in plant research, which highest starch content arose at a photoperiod of 12:12 h light/dark cycle (Smith, 2012), the duckweed starch content showed the different pattern. The reason might be due to the species difference between duckweed and Arabidopsis. The duckweed accumulated more starch than Arabidopsis, suggesting the different starch metabolism regulation mechanism in duckweed. Recently, the duckweed genome, transcriptome and proteome had been reported (Wang et al., 2014a,b; Tao et al., 2013; Huang et al., 2014). More interesting story will be explored in duckweed starch metabolism in the future. 
Table 2

Duckweed maximum starch content and maximum starch yield under three photoperiod cycles and six light intensities.

\begin{tabular}{lccc}
\hline $\begin{array}{l}\text { Photoperiod } \\
(\mathrm{L}: \mathrm{D} \text { cycle }) \mathrm{h}\end{array}$ & $\begin{array}{l}\text { Light intensity } \\
\left(\mu \mathrm{mol} \mathrm{m} \mathrm{m}^{-2} \mathrm{~s}^{-1}\right)\end{array}$ & $\begin{array}{l}\text { Maximum starch } \\
\text { content }(\%)\end{array}$ & $\begin{array}{l}\text { Maximum starch } \\
\text { yield }\left(\mathrm{g} \mathrm{m}^{-2}\right)\end{array}$ \\
\hline $12: 12$ & 400 & $56.36 \pm 2.60$ & $83.09 \pm 2.30$ \\
& 200 & $52.04 \pm 3.92$ & $43.83 \pm 3.55$ \\
& 110 & $42.16 \pm 3.69$ & $30.75 \pm 1.24$ \\
& 80 & $29.94 \pm 0.67$ & $20.05 \pm 1.07$ \\
& 50 & $13.60 \pm 0.37$ & $8.79 \pm 0.24$ \\
$16: 8$ & 20 & $5.29 \pm 0.20$ & $2.11 \pm 0.03$ \\
& 400 & $62.24 \pm 2.38$ & $81.33 \pm 2.96$ \\
& 200 & $57.94 \pm 1.89$ & $59.08 \pm 1.91$ \\
& 110 & $55.47 \pm 2.95$ & $52.25 \pm 1.39$ \\
& 80 & $43.52 \pm 0.70$ & $32.77 \pm 1.66$ \\
& $16.94 \pm 0.82$ & $9.53 \pm 1.07$ \\
& 50 & $3.86 \pm 0.18$ & $1.68 \pm 0.05$ \\
& 20 & $61.83 \pm 1.24$ & $54.66 \pm 3.33$ \\
& 400 & $62.71 \pm 3.54$ & $77.47 \pm 4.14$ \\
& 200 & $61.68 \pm 1.29$ & $98.70 \pm 4.63$ \\
& 110 & $56.49 \pm 1.18$ & $60.74 \pm 4.83$ \\
& 80 & $53.54 \pm 2.90$ & $39.09 \pm 1.72$ \\
& 50 & $6.82 \pm 1.21$ & $3.88 \pm 0.32$
\end{tabular}

Data are means of three repeated experiments and error bars indicate standard deviations.

In general, the duckweed starch content was increased during the 39 days cultivation. With the increased time of cultivation, the duckweed accumulated more and more biomass, which might result in the exhaust of nutrient in the culture medium, especially nitrogen and phosphorus. The dynamic variation of nitrogen and phosphorus in the culture medium during cultivation had been reported in our previously study (Yu et al., 2014). The starch accumulation induced by nutrient deficiency of nitrogen and phosphorus had been well investigated and reviewed in duckweed (Thorsteinsson and Tillberg, 1987; Xu et al., 2012; Cui and Cheng, 2015). Notably, we found that under long photoperiod (16:8 and 24:0) and high light ( 200 and $400 \mu \mathrm{mol} \mathrm{m}^{-2} \mathrm{~s}^{-1}$ ) with short time, duckweed can accumulate much more starch compared to other condition, while the duckweed biomass was not increased markedly (Fig. 2b and c). The results indicated that more fluxes of carbon source obtained by photosynthesis were used for starch accumulation, rather than respiration or reproduction in this condition. Besides widely using nutrient deficiency, high light induction may be regarded as another promising method for starch accumulation in future industrial large-scale duckweed cultivation. Nutrient deficiency accompanied by high light induction may be considered as a more effective method in duckweed starch accumulation. In other species, such as microalgae, high light and nitrogen deficiency as a powerful method to induce lipid accumulation had been widely applied (Ma et al., 2013). However, this method has not been widely used in duckweed starch accumulation. The reason may be that duckweed is cultivated in outdoor with sunlight as the only light resource at present (Xu et al., 2011; Xiao et al., 2013). Few industrial cultivation models with controlled light condition have been reported in duckweed cultivation. Taken together, these results suggested that high light induction could be considered as an effective method in starch accumulation for future industrial large-scale duckweed cultivation.

\subsection{Effect of light intensity and photoperiod on duckweed starch production}

Duckweed starch production is the most important consideration for application of duckweed in biofuel production. Starch production in duckweed mainly depended on not only starch content but also biomass production. In this study, the starch production of duckweed cultivated at three different photoperiods (12:12, 16:8 and 24:0 h light:dark) and six different light intensities (20, 50, $80,110,200$ and $400 \mu \mathrm{mol} \mathrm{m}^{-2} \mathrm{~s}^{-1}$ ) was calculated by starch content and biomass production. The results showed that the starch production was consistently increased during cultivation except at $400 \mu \mathrm{mol} \mathrm{m}^{-2} \mathrm{~s}^{-1}$ under a photoperiod of $24: 0 \mathrm{~h}$ light/dark cycle (Fig. 4). With increasing light intensity, the duckweed starch production was increased except at 200 and $400 \mu \mathrm{mol} \mathrm{m}^{-2} \mathrm{~s}^{-1}$ under a photoperiod of 24:0 h light/dark cycle (Fig. 4 and Table 2). As to the influence of photoperiod on starch production, the results showed that the duckweed starch production was increased with the extending photoperiod except at $400 \mu \mathrm{mol} \mathrm{m} \mathrm{m}^{-2} \mathrm{~s}^{-1}$ under a
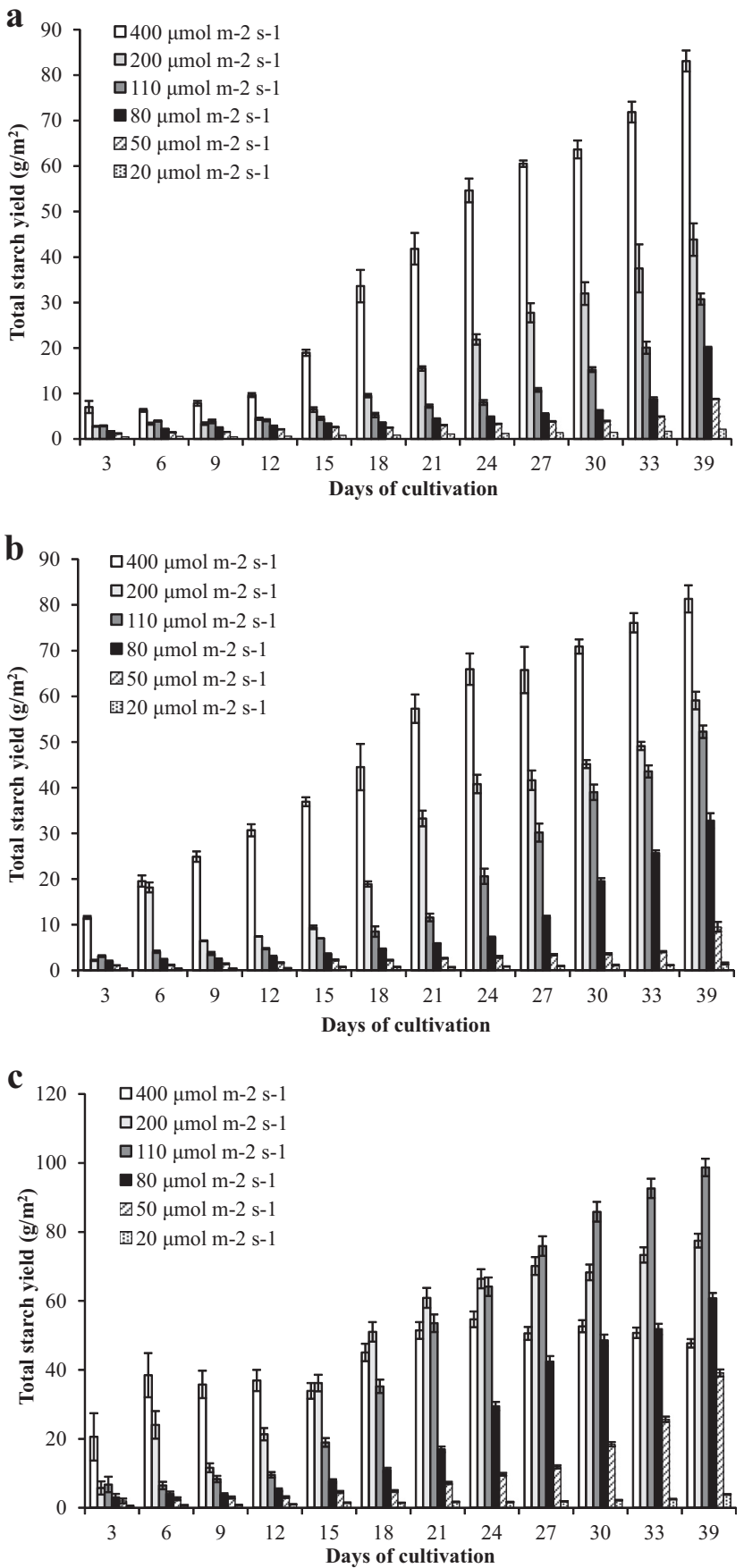

Fig. 4. Duckweed starch production under three different photoperiod cycles of 12:12 (a), 16:8 (b) and 24:0 (c) and six different light intensities $(20,50,80,110$, 200 and $400 \mu \mathrm{mol} \mathrm{m}^{-2} \mathrm{~s}^{-1}$ ). Data are means of three repeated experiments and error bars indicate standard deviations. 
photoperiod of $24: 0 \mathrm{~h}$ light/dark cycle (Fig. 4 and Table 2). Although the starch content was high, the decrease of biomass production at high light intensity and long photoperiod resulted in its low starch production (Fig. 2c, Figs. 3c and 4c). Notably, we found that the highest starch production was obtained at $110 \mu \mathrm{mol} \mathrm{m}^{-2}$ $\mathrm{s}^{-1}$ under a photoperiod of $24: 0 \mathrm{~h}$ light/dark cycle. In the future industrial duckweed cultivation, the results suggested that $110 \mu \mathrm{mol} \mathrm{m} \mathrm{m}^{-2} \mathrm{~s}^{-1}$ was the best choice for duckweed starch production. Similar with the starch content variation, high light intensity under long photoperiod especially at $400 \mu \mathrm{mol} \mathrm{m}^{-2} \mathrm{~s}^{-1}$ under a photoperiod of 24:0 h light/dark cycle also showed a rapid induction of starch production after 6-day cultivation. Therefore, high light induction could be considered as another effective method to increase duckweed starch accumulation for future industrial duckweed cultivation.

\section{Conclusions}

To investigate the effects of light conditions on duckweed biomass and starch production, $L$. aequinoctialis 6000 was cultivated at three photoperiods and six light intensities. The results showed that the duckweed growth and starch production was increased with increasing light intensity and photoperiod except at 200 and $400 \mu \mathrm{mol} \mathrm{m} \mathrm{m}^{-2} \mathrm{~s}^{-1}$. Considering the light cost, $110 \mu \mathrm{mol} \mathrm{m}{ }^{-2}-$ $\mathrm{s}^{-1}$ was the best light condition for duckweed biomass and starch production. Moreover, the results also suggested that high light induction was a promising method for duckweed starch production. This study provides optimized light conditions for future industrial large-scale duckweed cultivation.

\section{Acknowledgements}

This study was supported by the National Key Technology Research and Development Program of China (2015BAD15B01), Director Innovation Foundation of the Qingdao Institute of Bioenergy and Bioprocess Technology, and Shandong Agricultural Significant Application of Technology Innovation Program.

\section{References}

Appenroth, K.J., Borisjuk, N., Lam, E., 2013. Telling duckweed apart: genotyping technologies for the Lemnaceae. Chin. J. Appl. Environ. Biol. 19, 1-10.

Bergmann, B.A., Cheng, J., Classen, J., Stomp, A.M., 2000. In vitro selection of duckweed geographical isolates for potential use in swine lagoon effluent renovation. Bioresour. Technol. 73, 13-20.

Cheng, J.J., Stomp, A.M., 2009. Growing duckweed to recover nutrients from wastewaters and for production of fuel ethanol and animal feed. Clean-Soil Air Water 37, 17-26.

Cui, W.H., Cheng, J.J., 2015. Growing duckweed for biofuel production: a review. Plant Biol. 17, 16-23.

Cui, W.H., Xu, J.J., Cheng, J.J., Stomp, A.M., 2011. Starch accumulation in duckweed for bioethanol production. Biol. Eng. 3, 187-197.

Endo, A., Nakamura, T., Ando, A., Tokuyasu, K., Shima, J., 2008. Genome-wide screening of the genes required for tolerance to vanillin, which is a potential inhibitor of bioethanol fermentation, in Saccharomyces cerevisiae. Biotechnol. Biofuels 1, 1-6.

Ge, X.M., Zhang, N.N., Phillips, G.C., Xu, J.F., 2012. Growing Lemna minor in agricultural wastewater and converting the duckweed biomass to ethanol. Bioresour. Technol. 124, 485-488.
Huang, M.J., Fang, Y., Xiao, Y., Sun, J.L., Jin, Y.L., Tao, X., Ma, X.R., He, K.Z., Zhao, H., 2014. Proteomic analysis to investigate the high starch accumulation of duckweed (Landoltia punctata) under nutrient starvation. Ind. Crop Prod. 59, 299-308.

Im, C.S., Grossman, A.R., 2001. Identification and regulation of high light-induced genes in Chlamydomonas reinhardtii. Plant J. 30, 301-313.

Jackson, S.D., 2009. Plant responses to photoperiod. New Phytol. 181, 517-531.

Janas, K.M., Osiecka, R., 1995. Effect of aminophosphonates on starch accumulation in Spirodela punctata. Biol. Pharm. Bull. 32, 70-78.

Janas, K.M., Osiecka, R., Zon, J., 1998. Growth-retarding effect of 2-aminoindan-2phosphonic acid on Spirodela punctata. J. Plant Growth Regul. 17, 169-172.

Ma, Y.B., Wang, Z.Y., Zhu, M., Yu, C.J., Cao, Y.P., Zhang, D.Y., Zhou, G.K., 2013. Increased lipid productivity and TAG content in Nannochloropsis by heavy-ion irradiation mutagenesis. Bioresour. Technol. 136, 360-367.

McLaren, J.S., Smith, H., 1976. The effect of abscisic acid on growth, photosynthetic rate and carbohydrate metabolism in Lemna minor L. New Phytol. 76, 11-20.

Niyogi, K.K., 1999. Photoprotection revisited: genetic and molecular approaches. Annu. Rev. Plant Physiol. Plant Mol. Biol. 50, 333-359.

Peng, J.F., Wang, B.Z., Song, Y.H., Yuan, P., 2007. Modeling N transformation and removal in a duckweed pond: model development and calibration. Ecol. Model. 206, 147-152.

Reid, M.S., Bieleski, R.L., 1970. Response of Spirodela oligorrhiza to phosphorus deficiency. Plant Physiol. 46, 609-613.

Sánchez, Ó.J., Cardona, C.A., 2008. Trends in biotechnological production of fue ethanol from different feedstocks. Bioresour. Technol. 99, 5270-5295.

Sarkar, N., Ghosh, S.K., Bannerjee, S., Aikat, K., 2012. Bioethanol production from agricultural wastes: an overview. Renew. Energy 37, 19-27.

Schenk, R.U., Hildebrandt, A.C., 1972. Medium and techniques for induction and growth of monocotyle-donous and dicotyledonous plant cell cultures. Can. J. Bot. 50, 199-204.

Smith, A.M., 2012. Starch in the Arabidopsis plant. Starch 64, 421-434.

Smith, A.M., Zeeman, S.C., 2006. Quantification of starch in plant tissues. Nat. Protoc. $1,1342-1345$.

Stitt, M., Zeeman, S.C., 2012. Starch turnover: pathways, regulation and role in growth. Curr. Opin. Plant Biol. 15, 282-292.

Tao, X., Fang, Y., Xiao, Y., Jin, Y.L., Ma, X.R., Zhao, Y., He, K.Z., Zhao, H., Wang, H.Y. 2013. Comparative transcriptome analysis to investigate the high starch accumulation of duckweed (Landoltia punctata) under nutrient starvation. Biotechnol. Biofuels 6, 72 .

Thorsteinsson, B., Tillberg. J.E., 1987. Carbohydrate partitioning photosynthesis and growth in Lemna gibba G3. II. Effects of phosphorus limitation. Physiol. Plant. 71, 271-276.

Wang, W.Q., Haberer, G., Gundlach, H., Gläßer, C., Nussbaumer, T., Luo, M.C., Lomsadze, A., Borodovsky, M., Kerstetter, R.A., Shanklin, J., Byrant, D., Mockler, T., Appenroth, K.J., Grimwood, J., Jenkins, J., Chow, J., Choi, C., Adam, C., Cao, X.H., Fuchs, J., Schubert, I., Rokhsar, D., Schmutz, J., Michael, T.P., Mayer, K.F.X. Messing, J., 2014a. The Spirodela polyrhiza genome reveals insights into its neotenous reduction, fast growth, and aquatic lifestyle. Nat. Commun. 5, 3311.

Wang, W.Q., Wu, Y.R., Messing, J., 2014b. RNA-Seq transcriptome analysis of Spirodela dormancy without reproduction. BMC Genomics 15, 60.

Xiao, Y., Fang, Y., Jin, Y.L., Zhang, G.X., Zhao, H., 2013. Culturing duckweed in the field for starch accumulation. Ind. Crop Prod. 48, 183-190.

Xu, J.L., Shen, G.X., 2011. Growing duckweed in swine wastewater for nutrient recovery and biomass production. Bioresour. Technol. 102, 848-853.

Xu, J.L., Cui, W.H., Cheng, J.J., Stomp, A.M., 2011. Production of high-starch duckweed and its conversion to bioethanol. Biosyst. Eng. 110, 67-72.

Xu, J.L., Zhao, H., Stomp, A.M., Cheng, J.J., 2012. The production of duckweed as a source of biofuels. Biofuels 15 (3), 589-601.

Yu, C.J., Sun, C.J., Yu, L., Zhu, M., Xu, H., Zhao, J.S., Ma, Y.B., Zhou, G.K., 2014 Comparative analysis of duckweed cultivation with sewage water and SH media for production of fuel ethanol. PLoS ONE 9, e115023.

Yu, L., Yu, C.J., Zhu, M., Cao, Y.P., Yang, H.Y., Zhang, X., Ma, Y.B., Zhou, G.K., 2015. Structure analysis of galactoarabinan from duckweed. Carbohydr. Ploym. 117, $807-812$.

Zhang, L., Zhao, H., Gan, M.Z., Jin, Y.L., Gao, X.F., Chen, O., Guan, J.F., Wang, Z.Y., 2011. Application of simultaneous saccharification and fermentation (SSF) from viscosity reducing of raw sweet potato for bioethanol production at laboratory, pilot and industrial scales. Bioresour. Technol. 102, 4573-4579.

Zhao, Z., Shi, H.J., Liu, Y., Zhao, H., Su, H.F., Wang, M.L., Zhao, Y., 2014. The influence of duckweed species diversity on biomass productivity and nutrient removal efficiency in swine wastewater. Bioresour. Technol. 167, 383-389. 